\title{
On-farm starling populations and other environmental and management factors associated with the presence of cefotaxime and ciprofloxacin resistant $E$. coli among dairy cattle in Ohio
}

Genet A. Medhanie ${ }^{a}$, David L. Pearl ${ }^{\mathrm{a}}$, Scott A. McEwen ${ }^{\mathrm{a}}$, Michele T. Guerin ${ }^{\mathrm{a}}$, Claire M.

Jardine $^{b}$, Jennifer Schrock ${ }^{c}$, Jeffrey T. LeJeune ${ }^{c}$

${ }^{\mathrm{a}}$ Department of Population Medicine, Ontario Veterinary College, University of Guelph, Guelph, ON, N1G 2W1, Canada

${ }^{\mathrm{b}}$ Department of Pathobiology, Ontario Veterinary College, University of Guelph, Guelph, ON, N1G 2W1, Canada

${ }^{c}$ Food Animal and Health Research Program, Ohio Agricultural Research and Development Center, The Ohio State University, Wooster, OH, 44691, USA

*Corresponding Author. Tel.: +1 3176177724

E-mail address: gasmelas@uoguelph.ca (G.A. Medhanie).

\begin{abstract}
Wild birds that forage around livestock facilities have been implicated as vectors of antimicrobial resistant organisms. Although antimicrobial resistant bacteria have been isolated from European starlings (Sturnus vulgaris), their role in the dissemination of antimicrobial resistant elements in livestock facilities needs further investigation. To determine whether on-
\end{abstract}


farm starling density and other factors were associated with the presence of cefotaxime and ciprofloxacin resistant $E$. coli among dairy cows in Ohio, bovine fecal pats from 150 farms were tested for the presence of cefotaxime and ciprofloxacin resistant $E$. coli. Each farm was visited twice (during the summer and fall of 2007-2009). Multi-level logistic regression models with a random intercept to account for fecal pats collected within a specific visit to a farm were used to assess the associations. The percentage of samples with cefotaxime and ciprofloxacin resistant $E$. coli was $13.4 \%$ and $13.6 \%$, respectively. The percentage of farms having at least one sample testing positive for cefotaxime and ciprofloxacin resistant E. coli was $56.7 \%$ and $48.7 \%$, respectively. The odds of detecting cefotaxime and ciprofloxacin resistant $E$. coli in the samples was significantly higher in 2007 compared to 2008 and 2009, in fall compared to summer, and from farms closer than $60 \mathrm{~km}$ to starling night roost sites compared to the farms further than 60 $\mathrm{km}$. The presence of starlings during the day had a negative association with the likelihood of detecting cefotaxime resistant $E$. coli. Presence of calves also had a negative association with the likelihood of detecting both cefotaxime and ciprofloxacin resistant E. coli. European starlings might play a role in the dissemination of antimicrobial resistant agents in livestock facilities related to their daily population movements rather than the specific density of birds on farm during the day.

Keywords: cefotaxime, ciprofloxacin, E. coli, logistic regression, multi-level models, starling

\section{Introduction}

In addition to the risk of transmitting antimicrobial resistant organisms through animal products,

food animals can contaminate soil and water environments with resistant organisms that can also be transmitted to humans (Chee-Sanford et al., 2001). Wild birds that forage around livestock 
facilities have been implicated as vectors for the spread of antimicrobial resistant organisms (Dolejska et al., 2007; Literak et al., 2007; Bonnedahl and Jarhult, 2014). Birds that live close to livestock and human environments tend to carry a higher prevalence of antimicrobial resistant organisms compared to birds that live far from livestock and human surroundings (Allen et al., 2010). There is evidence suggesting that reciprocal inter-species transmission of antimicrobial resistance determinants is possible; similar sequence types of extended spectrum beta-lactamase producing Escherichia coli were isolated from Franklin's gulls (Leucophaeus pipixcan) and humans living in the same area (Hernandez et al., 2013).

European starlings (Sturnus vulgaris), one of the most abundant migratory bird species in North America (Feare, 1984), have been implicated in the transmission of zoonotic pathogens, such as E. coli O157:H7, Salmonella spp., and Campylobacter jejuni; (LeJeune et al., 2008; Williams et al., 2011; Carlson et al., 2012; Sanad et al., 2013; Carlson et al., 2015); however, their role in the transmission of antimicrobial resistant organisms needs further investigation. Ciprofloxacin resistant $C$. jejuni have been isolated from starlings captured on Ohio dairy cattle facilities (Sanad et al., 2013). Escherichia coli isolates from starlings captured on livestock facilities in Kansas were tested for their susceptibility to tetracycline, gentamicin, streptomycin, trimethoprim/sulphamethoxazole, kanamycin, chloramphenicol, and ampicillin, and 49\% showed resistance to at least one antimicrobial (Gaukler et al., 2009). Moreover, indistinguishable antimicrobial resistant Salmonella enterica isolates were recovered from bovine fecal and starling gastro-intestinal samples, supporting possible transmission of antimicrobial resistant $S$. enterica between cattle and starlings that live around dairy farms (Carlson et al., 2015). 
The distance of farms from starling night roost sites and on-farm starling density were found to be associated with the prevalence of E. coli O157:H7 in Ohio dairy cattle farms (Cernicchiaro et al., 2012; Swirski et al., 2014). In addition, statistically significant spatial clusters of molecular subtypes of E. coli $\mathrm{O} 157: \mathrm{H} 7$ isolated from bovine fecal pats, that were part of clades sharing cow and starling isolates, were detected on dairy farms close to night roost sites (Swirski et al., 2014). Further, spatial clusters of bovine fecal pats with a high prevalence of cefotaxime and ciprofloxacin resistant $E$. coli were identified around these same night roost sites (Medhanie et al., 2016). Therefore, the aim of this study was to determine if on-farm starling density and other management and environmental factors, including distance of farm from starling night roost sites, were associated with the presence of cefotaxime and ciprofloxacin resistant $E$. coli among dairy cattle in Ohio.

\section{Materials and Methods}

\subsection{Study farms and sampling}

Originally, samples were collected to study the association of wild bird density and farm management factors with the prevalence of $E$. coli O157:H7 (Cernicchiaro et al., 2012). Dairy farms $(\mathrm{n}=150)$ from 32 counties in Ohio, USA were selected from the Ohio Commercial Grade A

producers listing. Farms with at least 30 cows on a farm and willing to complete a questionnaire were included. Approximately $25 \mathrm{~g}$ of fresh fecal pats from the first 30 milking cows that were observed defecating, at the time of each visit, were collected and stored frozen. Each farm was visited twice (during the summer and fall of 2007-2009). Bovine fecal pat collection and questionnaire administration occurred during each visit and aliquots of the overnight nonselective broth enrichments of these fecal samples were stored at $-80^{\circ} \mathrm{C}$ in $30 \%$ buffered 
glycerol (Cernicchiaro et al., 2012). For the current study, five bovine fecal samples from each visit (for a total of 10 samples per farm) were tested for cefotaxime and ciprofloxacin resistant $E$. coli, except for two farms, for which samples were only available from the second visit; these stored samples were selected for testing based on the order they were collected from the farm on a particular visit (i.e., the first five of thirty samples collected from the farm on a particular visit were used for testing).

Using the data derived from the questionnaire previously administered to the study farms, we were able to gather information on temporal, environmental, and dairy farm management factors. Temporal factors included year (2007-2009) and season (summer (June-August) and fall (September-November)) of sampling. Environmental factors included on-farm starling density (i.e., number of starlings per milking cow), distance of farm from four starling night roost sites (Apple Creek, Lime Lakes, Morton, and South Rittman) (LeJeune et al., 2008), and presence of other mammals (wild/domestic) on the farm. Wild animals included raccoons, coyotes, deers, skunks, opossums, ground hogs, foxes and rabbits. Farm management factors included manure and feed management practices, and farm infrastructure (e.g., type of barn, number of barns, source of water for the cows, type of roof and ventilation) (Fig. 1). Training of bird observers that consisted of instructions on differentiating starlings from other avian species was performed in advance. On each visit, the number of starlings was counted on three areas of the farm (barns, feed storage, and manure storage) by one to three trained bird observers during the time of the day when starlings are most actively foraging on farms (08:30 to 16:40 h). Birds were counted for 5 minutes with 10 minute intervals (four times within an hour). The highest of the counts among the counting periods was recorded as the number of starlings on the farm (Cernicchiaro et al., 2012). Each recording of starling number from barn, feeding area and manure was recorded 
separately and added to the total number of starlings on farm. A full description of the

questionnaire has been reported previously (Cernicchiaro et al., 2012; Medhanie et al., 2015).

Formal sample-size calculations were not conducted at the start of this study because the data were collected for a previous study that assessed the association between starling density and other risk factors on the prevalence of $E$. coli $\mathrm{O} 157: \mathrm{H} 7$ in bovine fecal pats. However, a study in Ohio dairy farms detected blaCMY-2 carrying E. coli from $94.8 \%$ of bovine fecal samples (Mollenkopf et al., 2012). Assuming a 15\% lower prevalence of cefotaxime and ciprofloxacin resistant $E$. coli in samples from cattle on farms with a low starling density (compared to a high density), and an intra-class correlation coefficient of 0.8 (estimated using datum from Heider et al., 2009), four samples from 150 farms would be adequate to detect this difference with $80 \%$ power and 95\% confidence. Consequently, the collected sample size (five samples per visit) should have given us sufficient power to detect a difference in prevalence between the two groups.

\subsection{Antimicrobial resistance detection}

For each enriched fecal sample, a loopful (10 $\mu$ l) of the sample was streaked onto MacConkey agar supplemented with $2 \mu \mathrm{g} / \mathrm{ml}$ cefotaxime on one section and $1 \mu \mathrm{g} / \mathrm{ml}$ ciprofloxacin on another section, and incubated for 24 to 36 hours at $37^{\circ} \mathrm{C}$. Up to three lactose-positive (if present) colonies from each media were selected and confirmed as E. coli using the indole test. A sample was positive if at least one colony of $E$. coli was confirmed.

\subsection{Multi-level logistic regression model building}


Associations between the presence of cefotaxime and ciprofloxacin resistant $E$. coli in bovine fecal pats (the outcomes) with temporal, environmental, and farm management factors were assessed using multivariable multilevel logistic regression models with random intercepts to control for clustering of fecal pats nested within a specific visit to a farm and visits nested within farms. Initially, logistic regression models with one fixed effect and the random intercepts described above were fitted using the "melogit" command in Stata 13 (Stata Corporation, College Station, TX, USA) to screen associations using a liberal significance level of $\alpha=0.2$.

Potential collinearity among the independent variables, that were significant on univariable screening, was investigated using the Spearman rank correlation test and pairs of variables with rho $(\rho) \geq|0.8|$ were considered to be of concern. If variables were highly correlated, only one of them was selected for inclusion in the multivariable model based on biological plausibility or fewer missing observations. The linearity of continuous exposure variables against the logit of the outcome was assessed graphically using lowess curves. If the relationship was not linear the following were attempted: 1) a quadratic relationship was modeled if appropriate based on visualization of the relationship; 2) a log transformation was used to linearize the relationship; or 3) if 1 and 2 were not appropriate or successful, the variable was categorized based on quartiles or biologically relevant cut-points.

Each final model (cefotaxime resistance; ciprofloxacin resistance) was built using a manual backward elimination of non-significant variables at $\alpha=0.05$, unless the variables were confounders or part of a significant interaction term. Confounding was assessed following removal of a non-significant variable from the model by inspecting changes in the coefficients of the remaining significant variables. Changes $>25 \%$ were considered to indicate confounding 
assuming the variable was not an intervening variable. Interaction effects between pairs of the remaining exposure variables were generated and assessed in the main effects model. Any statistically significant interaction terms were retained in the model.

Pearson and deviance residuals at the fecal pat-level were assessed to identify potential outliers. The normality and homogeneity of variances of the best linear unbiased predictors (BLUPs) were assessed visually by examining normal quantile plots and scatter plots of BLUPS against the log odds of the predicted outcome, respectively. The variance partition coefficients were estimated from the variance components of these models using the latent variable technique (Dohoo et al., 2009).

\section{Results}

\subsection{Descriptive summary}

Bovine fecal samples $(\mathrm{n}=1,490)$ were tested for cefotaxime and ciprofloxacin resistant E. coli; 740 samples were from the first visit and 750 were from the second visit (Table 1). The yearly distribution of samples was 310, 540, and 640 in 2007, 2008, and 2009, respectively.

The proportion of samples with cefotaxime and ciprofloxacin resistant E. coli was $13.4 \%$ (95\% CI: $11.7-15.3$ and $13.6 \%$ (95\% CI: 11.9 - 15.4), respectively (Table 1), with $56.8 \%$ (95\% CI: 8.3- 64.7) of the farms testing positive for cefotaxime and $48.7 \%$ (95\% CI: 40. 4- 57.0) for

ciprofloxacin resistant $E$. coli. A farm was considered positive for resistant $E$. coli if at least one sample tested positive.

\subsection{Assessment of continuous variables for linearity}


The distances of each farm from the four starling night roost sites were highly correlated ( $\rho \geq$ 0.9). Consequently, a variable for "distance from the closest night roost" was generated. This variable was evaluated by categorizing distance into quartiles as well as by dichotomizing the variable into two categories (less than or equal to $60 \mathrm{~km}$ and greater than $60 \mathrm{~km}$ away) based on previous reports of starling daily travel distance between feeding sites and night roosts in the study location (Homan et al., 2010). On-farm number of birds (starlings and other wild birds) at the time of a visit was categorized into quartiles. On-farm number of starlings and on-farm starling density were dichotomized into two categories (zero and greater than zero) due to the large number (41\%) of observations for which no starlings were identified; however, these two variables were perfectly correlated $(\rho=1.00)$. On-farm number of starlings was selected for further analysis because of fewer missing observations (density could not be calculated for some farms due to missing data for the number of milking cows).

\subsection{Univariable multi-level models}

The random intercept for farm was removed from the multi-level models during univariable screening because the variance at the farm level was very low (i.e., $<1.4 \times 10^{-11}$ ). Consequently, only a random intercept to account for fecal pats collected within a specific visit to a farm were included in subsequent models.

The presence of cefotaxime resistant $E$. coli in bovine fecal pats was significantly $(\mathrm{P} \leq 0.2)$ associated with the following factors on univariable analysis: year and season of sampling; distance of each farm from the closest night roost; on-farm number of birds; on-farm number of starlings; presence of calves, heifers, and wild mammals on farm; stall bedding material; and type of feed for the cows (Supplementary Table). 
The presence of ciprofloxacin resistant $E$. coli in bovine fecal pats was significantly $(\mathrm{P} \leq 0.2)$ associated with the following factors on univariable analysis: year and season of sampling; distance of each farm from the closest night roost; on-farm number of birds; on-farm number of starlings; presence of calves and wild mammals on farm; stall bedding material; and type of feed, feeding method, and type of feed storage for the cows (Supplementary Table).

\subsection{Multivariable multi-level models}

The final multivariable models were similar for each outcome, except for on-farm number of starlings, which was significantly $(\mathrm{P} \leq 0.05)$ associated with the presence of cefotaxime resistant E. coli, but not ciprofloxacin resistant E. coli (Table 2). The odds of detecting cefotaxime resistant E. coli in bovine fecal pats was lower on farms in which starlings were present compared to farms in which starlings were absent.

Year and season of sampling, distance from the closest night roost, and presence of calves on farm were significantly associated with the presence of cefotaxime and ciprofloxacin resistant $E$. coli in bovine fecal pats (Table 2). The odds of detecting cefotaxime and ciprofloxacin resistant E. coli in fecal pats were lower in 2008 and 2009 compared to 2007. There was no significant difference between 2008 and 2009 for cefotaxime resistant $E$. coli $(\mathrm{OR}=1.35,95 \%$ CI: $0.51-$ $3.55 ; \mathrm{P}=0.541)$; however, for ciprofloxacin resistant $E$. coli, the odds were significantly higher in 2008 compared to 2009 ( $\mathrm{OR}=4.34,95 \%$ CI: $1.40-13.44 ; \mathrm{P}=0.011$ ). The odds of cefotaxime and ciprofloxacin resistant $E$. coli in the fecal pats were higher in fall than in summer. The distance of each farm from the closest night roost site was significant when modeled by quartile and as a dichotomous variable; the dichotomous variable is presented in the final models for simplicity (Table 2). The odds of cefotaxime and ciprofloxacin resistant E. coli 
were lower in fecal pats from farms that were further than $60 \mathrm{~km}$ to the nearest night roost compared to those closer than $60 \mathrm{~km}$. The presence of calves on farm at the time of sampling had a negative association with the likelihood of detecting cefotaxime and ciprofloxacin resistant $E$. coli.

Similar to the univariable models, the variance explained at the farm level was extremely low for both models $\left(1.1 \times 10^{-33}\right.$ and $1.0 \times 10^{-34}$ for the cefotaxime and ciprofloxacin models, respectively). Consequently, only the random intercept to account for fecal pats collected within a specific visit to a farm was included in each final model. The proportion of variance in the cefotaxime and ciprofloxacin resistance models that was explained at the level of a specific visit to a farm was $50 \%$ and $56 \%$, respectively. There were no concerning residuals or potential outliers identified by evaluation of the residuals and BLUPs; the BLUPs based on visual assessment were normally distributed and had a constant variance.

\section{Discussion}

Wild birds have been identified as potential reservoirs and vectors of antimicrobial resistant organisms (Smith et al., 2014; Carroll et al., 2015). Consequently, there is a need to investigate the role of birds in spreading resistant genetic elements in bacteria to livestock and human environments. This study investigated the potential role of European starlings in the transmission of antimicrobial resistant organisms among dairy cattle farms. Variables related to year, season, starling populations, and farm management were significantly associated with the presence of cefotaxime and ciprofloxacin resistant $E$. coli in bovine fecal pats. 
We found that the odds of detecting cefotaxime and ciprofloxacin resistant E. coli in bovine fecal pats were lower in 2008 and 2009 compared to 2007. Although the same farms were not repeatedly sampled every year, year was also associated with starling density in our previous study of management factors that influence on-farm density of European starlings in Ohio, which utilized the same dataset (Medhanie et al., 2015). In addition, there has been anecdotal information from farmers that participated in the study that starlings had become less of a problem over the study period. These annual changes might reflect broader changes in the starling population throughout the region. Seasonal changes might relate to the nature of the foraging behaviour of the birds. During the late spring and early summer, which is the starling's breeding season, starlings are less active on farms while they forage for insects for their young, whereas in late summer and fall they more actively forage on livestock feed (Kessel, 1957; Williamson and Gray, 1975).

Distance between farm and the closest night roost site was significantly associated with the presence of cefotaxime and ciprofloxacin resistant $E$. coli in the multivariable models. This finding is consistent with a previous analysis based on E. coli O157:H7 (Swirski et al., 2014), and is supported by our previous finding of significant spatial clusters of bovine fecal pats with a high prevalence of cefotaxime and ciprofloxacin resistant $E$. coli around these same night roost sites (Medhanie et al., 2016). Even after adjusting for on-farm starling density in the spatial analysis, there were statistically significant clusters of cefotaxime and ciprofloxacin resistant $E$. coli around these night roosts, suggesting that the clusters of antimicrobial resistant isolates do not depend on the specific density of starlings during the day, but possibly the movement of starlings on farms during travel to and from night roosts (Medhanie et al., 2016). In our ciprofloxacin model, the on-farm number of starlings was not a statistically significant variable, 
yet in our cefotaxime model, the presence of starlings on farm had a negative association with the presence of cefotaxime resistant $E$. coli in bovine fecal pats. The latter finding is in contrast with a previous finding that starling density was positively associated with E. coli $\mathrm{O} 157: \mathrm{H} 7$ (Cernicchiaro et al., 2012). Our finding needs to be further investigated in terms of starling feeding and roosting habit. It is possible that the protective effect of presence of starlings was due to chance (i.e., a type I error), but also starling numbers may be a proxy for other variables related to biosecurity and/or choice of antimicrobials used on farm.

The protective effect of having calves on a farm for the presence of cefotaxime and ciprofloxacin resistant $E$. coli might reflect differences in biosecurity between farms. Acquiring or raising replacement heifers offsite, where animals are mixed from multiple farms might allow for the introduction of resistant organisms when these heifers are reintroduced to a herd. Maintaining a closed herd has been recommended as a biosecurity measure against other cattle pathogens and could also protect against the introduction of antimicrobial resistant organisms (Wells, 2000). Further study of replacement heifers and their management is warranted.

Initially, the hierarchical structure of our models included fecal samples, visit (capturing location and time of sampling), and the farm itself. The extremely small proportion of variance explained by the farm level suggests that the correlation in the prevalence of resistance was very small between visits to the same farm. However, the large variance component at the visit level for a particular farm indicates that the correlation among samples in terms of carrying resistant E. coli at the time of a visit was relatively high. This finding indicates that farm management and environmental factors that vary on a farm by visit or season (summer vs. fall) could be very important in terms of the prevalence of resistant E.coli on Ohio dairy farms. 
This study made use of the stored, enriched fecal samples collected as part of another study on $E$. coli $\mathrm{O} 157: \mathrm{H7}$; consequently, on-farm antimicrobial usage was not recorded. However, other studies have documented the frequent usage of antimicrobials on Ohio dairy farms (Tragesser et al., 2006; Heider et al., 2009). Ceftiofur (a third generation cephalosporin) was used by $88 \%$ of the dairy farms in a survey that recorded antimicrobial usage in this area in 2009 (Heider et al., 2009). In that study, the usage of ceftiofur was not associated with the detection of $E$. coli resistance to ceftriaxone. On-farm use might be less relevant when antimicrobial resistant organisms are readily dispersed in the environment and overall prevalence of antimicrobial usage is particularly high.

\section{Conclusion}

Based on the associations observed, European starling night roost sites could be sources of antimicrobial resistant organisms to dairy cattle farms in Ohio. In particular, large changes in populations of starlings and their movements throughout the landscape might be more important than the actual number of birds observed on farm. Consequently, additional studies in other regions are required to confirm the importance of the impact of these birds throughout the environment and assess the impact of limiting bird access to farms.

\section{Acknowledgements}

This research was funded by the United States Department of Agriculture through their National Research Initiative (USDA-NRI), Epidemiological Approaches to Food Safety Grant \# 200601227. Computational infrastructure was available through a grant to D. L. Pearl from the Canada Foundation for Innovation and the Ontario Ministry of Research and Innovation. We 
thank Mrs. Pamela Schlegel for her valuable consultation on dairy cattle farm management practices in Ohio.

\section{References}

Allen, H.K., Donato, J., Wang, H.H., Cloud-Hansen, K.A., Davies, J., Handelsman, J., 2010.

Call of the wild: antibiotic resistance genes in natural environments. Nat. Rev. Microbiol. 8, 251259.

Bonnedahl, J., Jarhult, J.D., 2014. Antibiotic resistance in wild birds. Ups. J. Med. Sci. 119, 113116.

Carlson, J.C., Ellis, J.W., Tupper, S.K., Franklin, A.B., Linz, G.M., 2012. The effect of European starlings and ambient air temperature on Salmonella enterica contamination within cattle feed bunks. Hum.Wildl. Interact. 6, 64-71.

Carlson, J.C., Hyatt, D.R., Ellis, J.W., Pipkin, D.R., Mangan, A.M., Russell, M., Bolte, D.S., Engeman, R.M., DeLiberto, T.J., Linz, G.M., 2015. Mechanisms of antimicrobial resistant Salmonella enterica transmission associated with starling-livestock interactions. Vet. Microbiol. $179,60-68$.

Carroll, D., Wang, J., Fanning, S., McMahon, B.J., 2015. Antimicrobial resistance in wildlife: implications for public health. Zoonoses Public Health 62, 534-542. 
Cernicchiaro, N., Pearl, D.L., McEwen, S.A., Harpster, L., Homan, H.J., Linz, G.M., LeJeune, J.T., 2012. Association of wild bird density and farm management factors with the prevalence of E. coli $\mathrm{O} 157$ in dairy herds in Ohio (2007-2009). Zoonoses Public Health 59, 320-329.

Chee-Sanford, J.C., Aminov, R.I., Krapac, I.J., Garrigues-Jeanjean, N., Mackie, R.I., 2001. Occurrence and diversity of tetracycline resistance genes in lagoons and groundwater underlying two swine production facilities. Appl. Environ. Microbiol. 67, 1494-1502.

Dohoo, I., Martin, W., Stryhn, H., 2009. Veterinary Epidemiologic Research. VER Inc. PEI, Charlottetown, Canada.

Dolejska, M., Cizek, A., Literak, I., 2007. High prevalence of antimicrobial-resistant genes and integrons in Escherichia coli isolates from Black-headed Gulls in the Czech Republic. J. Appl. Microbiol. 103, 11-19.

Feare, C.J., 1984. The starling. Oxford University Press, Oxford, New york.

Gaukler, S.M., Linz, G.M., Sherwood, J.S., Dyer, N.W., Bleier, W.J., Wannemuehler, Y.M., Nolan, L.K., Logue, C.M., 2009. Escherichia coli, Salmonella, and Mycobacterium avium subsp paratuberculosis in wild European starlings at a Kansas cattle feedlot. Avian. Dis. 53, 544-551.

Heider, L.C., Funk, J.A., Hoet, A.E., Meiring, R.W., Gebreyes, W.A., Wittum, T.E., 2009. Identification of Escherichia coli and Salmonella enterica organisms with reduced susceptibility to ceftriaxone from fecal samples of cows in dairy herds. Am. J. Vet. Res. 70, 389-393.

Hernandez, J., Johansson, A., Stedt, J., Bengtsson, S., Porczak, A., Granholm, S., GonzalezAcuna, D., Olsen, B., Bonnedahl, J., Drobni, M., 2013. Characterization and comparison of 
extended-spectrum beta-lactamase (ESBL) resistance genotypes and population structure of Escherichia coli isolated from Franklin's gulls (Leucophaeus pipixcan) and humans in Chile. PLoS One 8, e76150.

Homan, H.J., Slowik, A.A., Penry, L.B., Linz, G.M., Bodenchuk, M.J., Gilliland, R.L., 2010. Site use of European starlings captured and radio-tagged at Texas feedlots during winter. Proc. 24th Vertebr. Pest Conf. Univ. of Calif., Davis, 250-256.

Kessel, B., 1957. A study of the breeding biology of the European starling (Sturnus vulgaris L.) in North America. Am. Midl. Nat. 58, 257-331.

LeJeune, J., Homan, J., Linz, G., Pearl, D.L., 2008. Role of the European starling in the transmission of E. coli O157 on dairy farms. Proc. 23rd Vertebr. Pest Conf. Univ. of Calif, Davis, pp. 31-34.

Literak, I., Vanko, R., Dolejska, M., Cizek, A., Karpiskova, R., 2007. Antibiotic resistant Escherichia coli and Salmonella in Russian rooks (Corvus frugilegus) wintering in the Czech Republic. Lett. Appl. Microbiol. 45, 616-621.

Medhanie, G.A., Pearl, D.L., McEwen, S.A., Guerin, M.T., Jardine, C.M., LeJeune, J.T., 2015. Dairy cattle management factors that influence on-farm density of European starlings in Ohio, 2007-2009. Prev. Vet. Med. 120, 162-168.

Medhanie, G.A., Pearl, D.L., McEwen, S.A., Guerin, M.T., Jardine, C.M., Schrock, J., LeJeune, J.T., 2016. Spatial clustering of cefotaxime and ciprofloxacin with reduced susceptibility to $E$. 
coli among dairy cattle relative to the European starling night roosts. Zoonoses Public Health. doi: 10.1111/zph.12296. [Epub ahead of print]

Mollenkopf, D.F., Weeman, M.F., Daniels, J.B., Abley, M.J., Mathews, J.L., Gebreyes, W.A., Wittum, T.E., 2012. Variable within- and between-herd diversity of CTX-M cephalosporinasebearing Escherichia coli isolates from dairy cattle. Appl. Environ. Microbiol. 78, 4552-4560.

Sanad, Y.M., Closs, G., Jr., Kumar, A., Lejeune, J.T., Rajashekara, G., 2013. Molecular epidemiology and public health relevance of Campylobacter isolated from dairy cattle and European starlings in Ohio, USA. Foodborne Pathog. Dis. 10, 229-236.

Smith, S., Wang, J., Fanning, S., McMahon, B.J., 2014. Antimicrobial resistant bacteria in wild mammals and birds: a coincidence or cause for concern? Irish Vet. J. 67, 8-8.

Swirski, A.L., Pearl, D.L., Williams, M.L., Homan, H.J., Linz, G.M., Cernicchiaro, N., LeJeune, J.T., 2014. Spatial epidemiology of Escherichia coli O157:H7 in dairy Cattle in relation to night roosts of Sturnus vulgaris (European Starling) in Ohio, USA (2007-2009). Zoonoses Public Health 61, 427-435.

Tragesser, L.A., Wittum, T.E., Funk, J.A., Winokur, P.L., Rajala-Schultz, P.J., 2006. Association between ceftiofur use and isolation of Escherichia coli with reduced susceptibility to ceftriaxone from fecal samples of dairy cows. Am. J. Vet. Res. 67, 1696-1700.

Wells, S. J., 2000. Biosecurity on dairy operations: hazards and risks. J. Dairy Sci. 83, 23802386. 
Williams, M.L., Pearl, D.L., LeJeune, J.T., 2011. Multiple-locus variable-nucleotide tandem repeat subtype analysis implicates European starlings as biological vectors for Escherichia coli O157:H7 in Ohio, USA. J. Appl. Microbiol. 111, 982-988.

Williamson, P., Gray, L., 1975. Foraging behavior of starling (Sturnus vulgaris) in Maryland. Condor 77, 84-89. 
1 Table 1. Sample and farm prevalence of cefotaxime and ciprofloxacin resistant E. coli

2 among Ohio dairy cattle (2007 - 2009)

\begin{tabular}{lllll}
\hline Antimicrobials & Visit 1 & Visit 2 & Visits 1 \& 2 & Farm \\
& $\mathbf{n}^{\mathbf{a}=\mathbf{7 4 0}}$ & $\mathbf{n}^{\mathbf{a}}=\mathbf{7 5 0}$ & $\mathbf{n}^{\mathbf{a}}=\mathbf{1 , 4 9 0}$ & $\mathbf{n}^{\mathbf{c}}=\mathbf{1 5 0}$ \\
\hline & $\mathbf{n}^{\mathbf{b}(\boldsymbol{\%})}$ & $\mathbf{n}^{\mathbf{b}}(\boldsymbol{\%})$ & $\mathbf{n}^{\mathbf{b}}(\boldsymbol{\%})$ & $\mathbf{n}^{\mathbf{b}}(\boldsymbol{\%})$ \\
\cline { 2 - 5 } Cefotaxime & $48(6.5)$ & $152(20.3)$ & $200(13.4)$ & $85(56.7)$ \\
Ciprofloxacin & $58(7.8)$ & $144(19.2)$ & $202(13.6)$ & $73(48.7)$ \\
Cefotaxime \& ciprofloxacin & $26(3.5)$ & $90(12.0)$ & $116(7.8)$ & $51(34.0)$ \\
\hline
\end{tabular}

3 a Number of samples

$4 \quad{ }^{b}$ Number of positive samples

$5 \quad{ }^{\mathrm{c}}$ A farm was considered positive for resistant E. coli if at least one sample tested positive. 
Table 2. Results of multivariable multi-level logistic regression models with a random intercept to account for fecal pats collected within a specific visit to a farm examining the association between temporal, environmental, and farm management factors with the presence of cefotaxime and ciprofloxacin resistant $E$. coli on Ohio dairy cattle farms $(n=$ 150 farms).

\begin{tabular}{|c|c|c|c|c|c|c|}
\hline \multicolumn{4}{|c|}{ Cefotaxime } & \multicolumn{3}{|c|}{ Ciprofloxacin } \\
\hline Variable & OR & $95 \% \mathrm{CI}$ & P-value & OR & $95 \% \mathrm{CI}$ & P-value \\
\hline \multicolumn{7}{|l|}{ Year } \\
\hline 2007 & $\operatorname{Ref}^{a}$ & & & $\operatorname{Ref}^{a}$ & & \\
\hline 2008 & 0.18 & $0.08,0.42$ & $<0.001$ & 0.22 & $0.09,0.53$ & 0.001 \\
\hline 2009 & 0.13 & $0.05,0.40$ & $<0.001$ & 0.05 & $0.01,0.19$ & $<0.001$ \\
\hline \multicolumn{7}{|l|}{ Season } \\
\hline Summer & $\operatorname{Ref}^{a}$ & & & $\operatorname{Ref}^{a}$ & & \\
\hline Fall & 4.07 & $2.01,8.21$ & $<0.001$ & 3.71 & $1.71,8.02$ & 0.001 \\
\hline $\begin{array}{l}\text { Distance } \\
(\mathbf{k m})\end{array}$ & & & & & & \\
\hline$\leq 60 \mathrm{~km}$ & $\operatorname{Ref}^{a}$ & & & $\operatorname{Ref}^{a}$ & & \\
\hline$>60 \mathrm{~km}$ & 0.31 & $0.11,0.83$ & 0.020 & 0.28 & $0.09,0.89$ & 0.032 \\
\hline
\end{tabular}




\begin{tabular}{|c|c|c|c|c|c|c|}
\hline \multicolumn{4}{|c|}{ Cefotaxime } & \multicolumn{3}{|c|}{ Ciprofloxacin } \\
\hline Variable & $\mathbf{O R}$ & $95 \% \mathrm{CI}$ & P-value & $\mathbf{O R}$ & $95 \% \mathrm{CI}$ & P-value \\
\hline \multicolumn{7}{|c|}{ On-farm no. of starlings } \\
\hline Absence of starlings & $\operatorname{Ref}^{\mathrm{a}}$ & & & $\operatorname{Ref}^{\mathrm{a}}$ & & \\
\hline Presence of starlings & 0.46 & $0.24,0.90$ & 0.023 & 0.58 & $0.28,1.20$ & 0.139 \\
\hline \multicolumn{7}{|l|}{ Calves on farm } \\
\hline No & $\operatorname{Ref}^{\mathrm{a}}$ & & & $\operatorname{Ref}^{\mathrm{a}}$ & & \\
\hline \multirow[t]{2}{*}{ Yes } & 0.23 & $0.10,0.49$ & $<0.001$ & 0.19 & $0.08,0.47$ & $<0.001$ \\
\hline & \multicolumn{6}{|c|}{ Variance components } \\
\hline Variance $(95 \%$ CI) & \multicolumn{3}{|c|}{$3.33(2.08,5.31)$} & \multicolumn{3}{|c|}{$4.23(2.63,6.83)$} \\
\hline $\operatorname{VPC}^{b}(95 \% \mathrm{CI})$ & \multicolumn{3}{|c|}{$0.50(0.39,0.62)$} & \multicolumn{3}{|c|}{$0.56(0.44,0.67)$} \\
\hline
\end{tabular}

\footnotetext{
${ }^{\mathrm{a}}$ Referent

${ }^{\mathrm{b}}$ Variance partition coefficient
} 


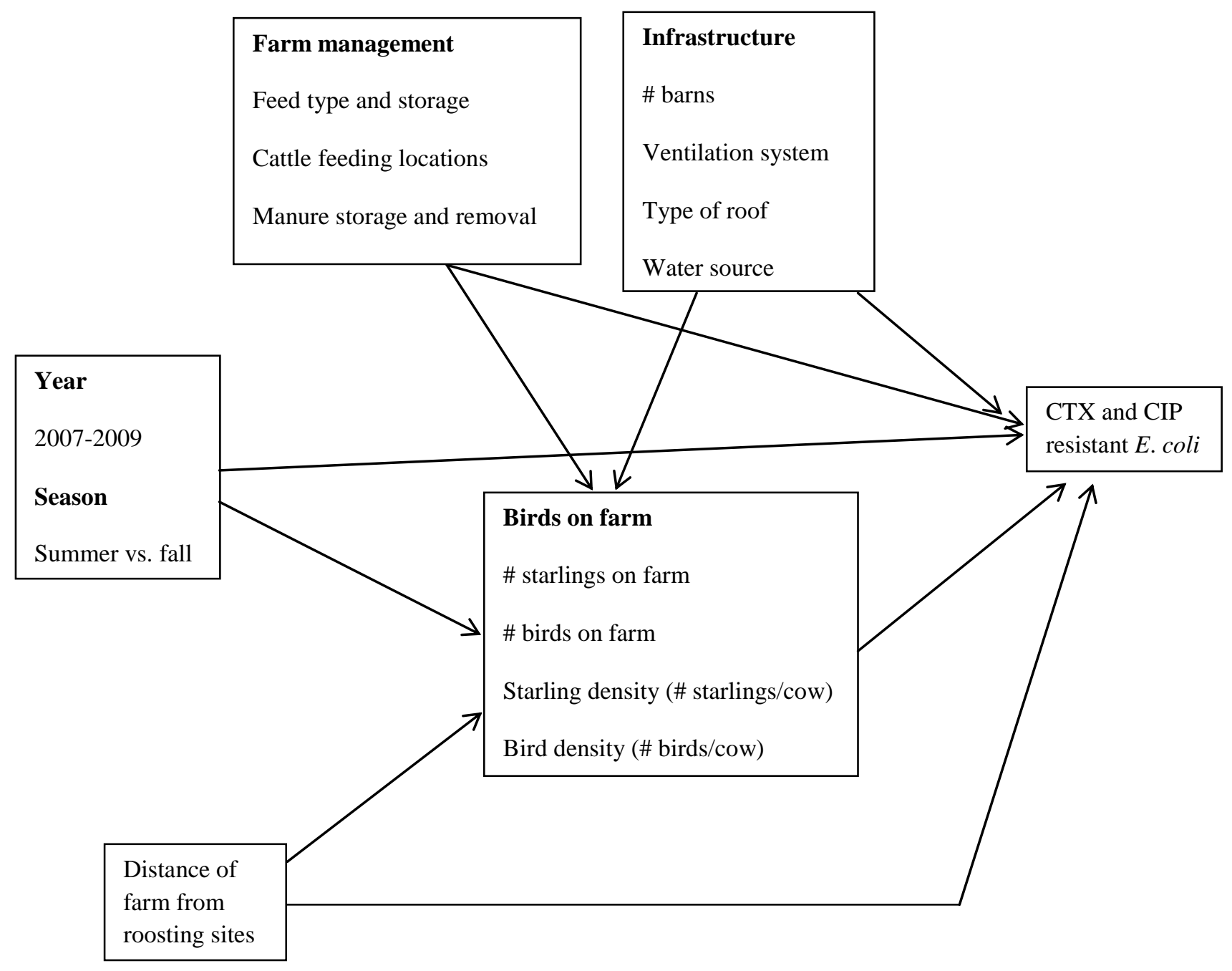

